\title{
Versatile fabrication of PDMS-carbon electrodes for silicone dielectric elastomer transducers
}

\section{O. Araromi; S. Rosset; H. Shea}

(C) 2015 IEEE. Personal use of this material is permitted. Permission from IEEE must be obtained for all other users, including reprinting/ republishing this material for advertising or promotional purposes, creating new collective works for resale or redistribution to servers or lists, or reuse of any copyrighted components of this work in other works. To access the final edited and published work see http://doi.org/10.1109/TRANSDUCERS.2015.7181323. 


\title{
VERSATILE FABRICATION OF PDMS-CARBON ELECTRODES FOR SILICONE DIELECTRIC ELASTOMER TRANSDUCERS
}

\author{
O. A. Araromi, S. Rosset, and H. R. Shea \\ Microsystems for Space Technologies Laboratory (LMTS), École Polytechnique Fédérale de Lausanne \\ (EPFL), Neuchâtel, Switzerland
}

\begin{abstract}
We report a novel method for the fabrication of polydimethylsiloxane (PDMS)-carbon composite electrodes for silicone dielectric elastomer transducers. The methodology combines patterning by laser ablation and oxygen plasma induced bonding, producing stretchable and flexible devices with exceptional electrode adhesion and high mechanical robustness. The methodology also offers great flexibility in the size and scale of electrode designs; we demonstrate this in the fabrication of flexible bending actuators $>10 \mathrm{~cm}$ long and interdigitated capacitive sensors with micro-scale features.
\end{abstract}

\section{KEYWORDS}

Dielectric elastomer actuators, dielectric elastomer sensor, stretchable devices, flexible bending actuators.

\section{INTRODUCTION}

Dielectric elastomers transducers (DETs) are a versatile and promising technology offering truly stretchable actuators and sensors [1]. Silicone elastomers are a popular dielectric material used in DET fabrication due to their relatively low viscous properties, enabling fast operation [2], and in the case of poly(dimethylsiloxane) (PDMS), optical transparency.

An important aspect in the development of DETs is the realization of compliant electrodes, allowing the highly elastic properties of the elastomer to be exploited. Many methods have been developed for the fabrication of electrodes for DETs, including carbon or graphite powers, greases or elastomer composites, implantation of metal ions and hydrogels filled with ionic solutions $[3,4]$. The carbon powder methods are particularly attractive due to their relatively low-cost, conductivity even at high strains ( $>$ $100 \%$ ) and generally low stiffening impact, which is an important consideration for making dielectric elastomer actuators. In particular, carbon powders in elastomer matrices offer good mechanical robustness due to chemical bonding between the electrode composite and the underlying elastomer membrane. Patterning of carbonelastomer electrodes can be realized with several methods including, stamp printing or spray deposition [5]. However, these methods can be limited in terms of pattern resolution, repeatability, adhesion or design flexibility.

Our technique involves patterning of electrodes by laser ablation and offers high repeatability, whilst being able to pattern micro-sized features. Combining with oxygen plasma bonding, a well-known technique used in micro-

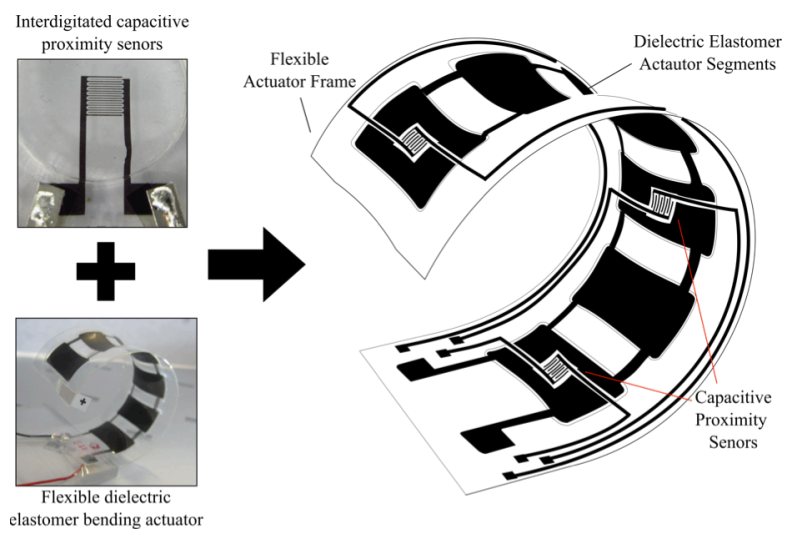

Figure 1: Concept design for a multifunctional flexible bending actuator with integrated capacitive sensors for contact-proximity detection.

fluidics for the bonding of polydimethylsiloxane (PDMS) to various substrates, our technique produces electrodes with high adhesion. Casting the uncured electrode by blade casting enables fabrication of electrodes of a variety of sizes (up to A4 paper size) and thicknesses, thereby allowing the resistance to be tuned as desired.

We fabricate flexible dielectric elastomer bending actuators of length $>10 \mathrm{~cm}$ and interdigitated capacitive proximity sensors with features $<100 \mu \mathrm{m}$ using our technique, demonstrating it's efficacy and versatility. This lays the foundation for the development of novel multifunction flexible robotic manipulators with integrated sensing capabilities fabricated with a single process flow (Figure 1). Such devices would be able to perform gripping functions whilst simultaneously providing sensory feedback about the proximity to the object being grasped.

\section{FABRICATION METHODOLOGY}

Our electrode material is made by mixing carbon black (Ketjenblack EC-300J, AkzoNobel) and a soft, highly elastic PDMS (MED-4901, NuSil) at a ratio of $1: 10$ by weight. The mixture is thinned with solvents to facilitate casting.

The electrode mixture is cast using a film applicator coater (Zehntner) to a thickness between $1 \mu \mathrm{m}$ up to several hundred micrometers onto a PET foil substrate coated with a poly(vinyl alcohol) (PVA) water soluble sacrificial layer (Figure 2i)) (The PDMS membrane is also cast in the same way, Figure 2ii)). The electrode is then cured in an oven at $80^{\circ} \mathrm{C}$ for 2 hours. The cured electrode is patterned by laser ablation using a $\mathrm{CO}_{2}$ laser engraver (Speedy 300, Trotec) 


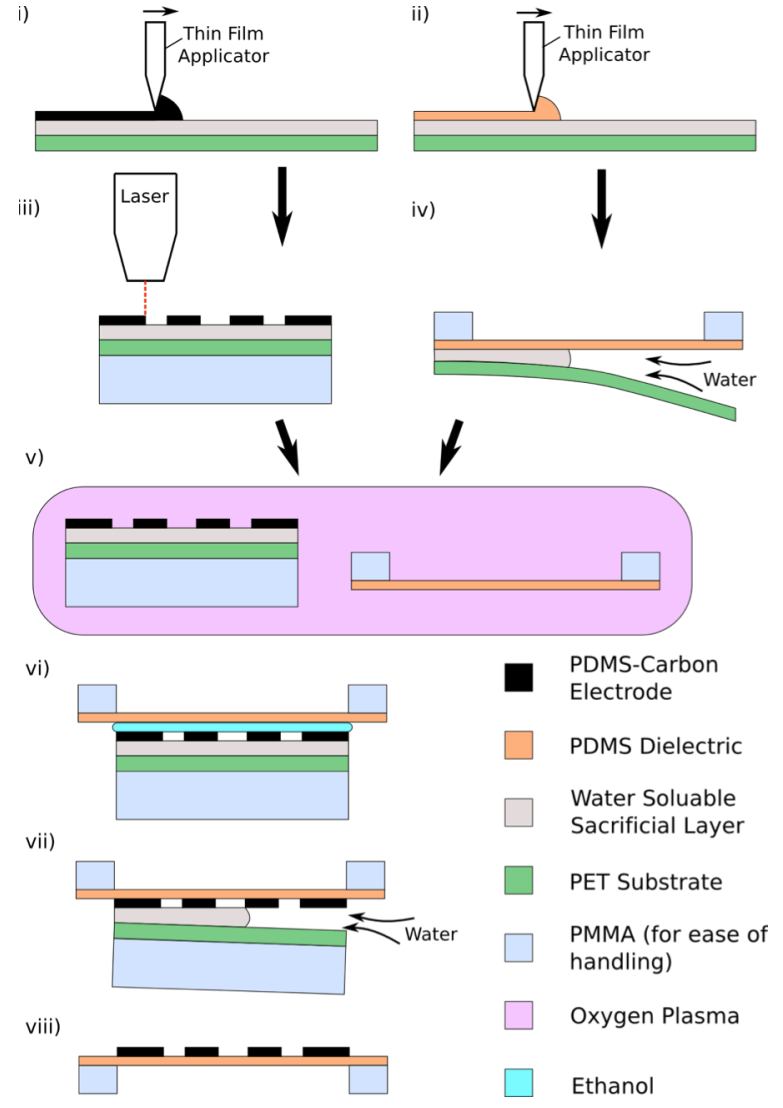

Figure 2: Process flow for our fabrication methodology

(Figure 2iii)). A $3 \mathrm{~mm}$ plastic (poly(methyl methacrylate) (PMMA)) support is bonded to the back of the PET electrode substrate to keep the electrode flat and to facilitate handing.

The PDMS dielectric is released from the PET substrate by submerging in hot water, dissolving the sacrificial layer (Figure 2iv)). The patterned electrode and PDMS membrane are treated in oxygen plasma at a power of $20 \mathrm{~W}$ for approximately 20 seconds (Figure 2v)), producing silanol groups on the exposed surfaces [6]. After treatment a small amount of ethanol is placed on the activated PDMS surface and the electrode is placed on the ethanol (Figure 2vi). As the ethanol evaporates, the two surfaces are brought into conformal contact forming covalent bonds as a result of condensation reaction of the silanol groups.

After 30 minutes the electrode is released by dissolving the sacrificial layer by submersion in hot water (Figure 2vii).

\section{Fabrication remarks}

Bonding repeatability: We observed that repeatable bonding was achieved when at least one of the bonding surfaces (typically the PDMS surface for our process) was unconstrained, that is freestanding and thus allowed to bend substantially. We believe this to be due to the ability of the unconstrained surface to naturally conform to the other bonding surface as the ethanol evaporates due to surface tension and electrostatic attraction. This conformal contact is necessary as the electrode and PDMS membrane are not perfectly flat (Figure 3C). When both surfaces are constrained by rigid structures conformal contact is inhibited due to the increased bending stiffness.

Bonding of electrodes with micro-sized features: Repeatable bonding of electrodes with micro-sized features $(<300 \mu \mathrm{m})$ was achieved on thin $(<10 \mu \mathrm{m})$ PDMS membranes but was less repeatable for thicker membranes. We believe this to be the result of the increased bending stiffness in the thicker elastomer membranes, which inhibits conformal contact of the membrane to small features (Figure 3B). We were able to circumvent this issue by bonding the electrode pattern first to a thin PMDS membrane and subsequently bonding the thin membrane to a thicker membrane. An alternative method is to apply pressure via a conformal object such as a water balloon.

Pattern resolution: With our laser ablation system, electrode features as small as $80 \mu \mathrm{m}$ could be achieved. Feature sizes on the scale of $1 \mu \mathrm{m}$ could potentially be realized with a higher resolution machine, such as a femtosecond laser [7].

Plasma induced stiffening: Exposing PDMS in oxygen plasma is known to have a stiffening effect on the PDMS
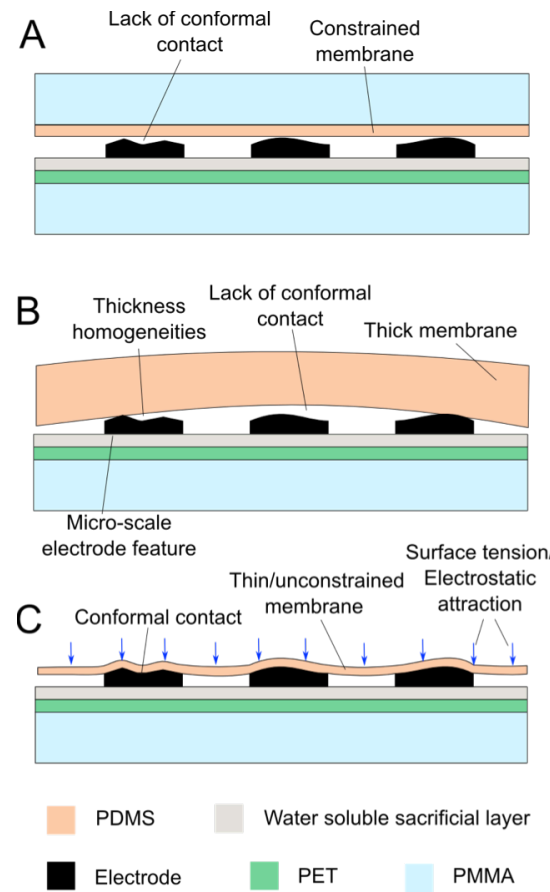

Figure 3: Schematic showing the difference in conformality between constrained, thick and thin/unconstrained PDMS membranes. A) Constrained PDMS membrane does not conform to the electrode. B) Thick membrane has a relatively large bending stiffness and can not easily conform to electrode micro features. C) Thin PDMS membrane more easily conforms to membrane via electrostatic attraction/surface tension. 

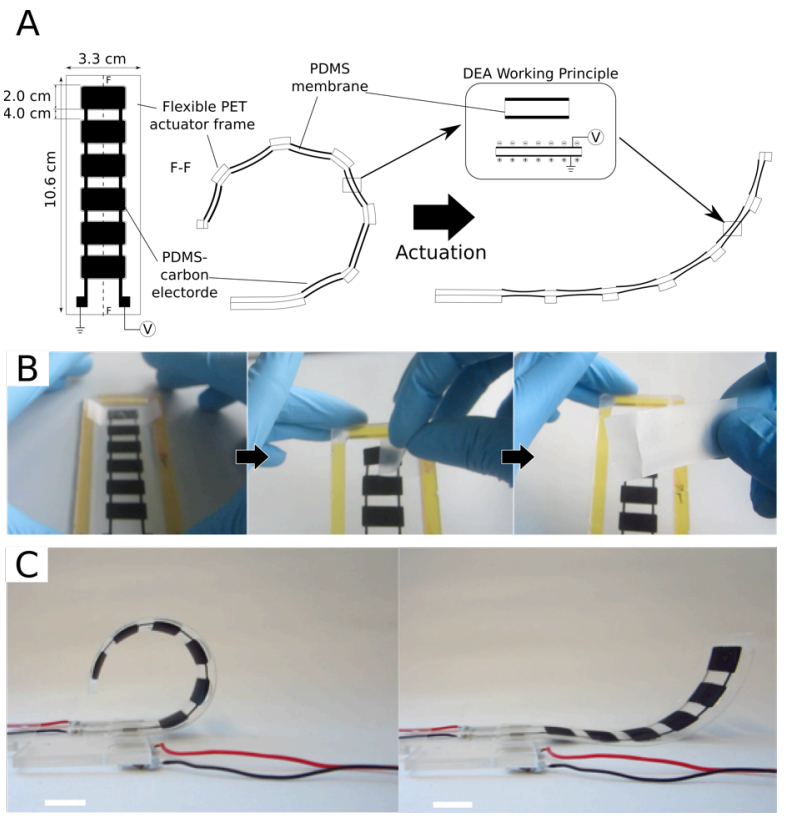

Figure 4: A $10 \mathrm{~cm}$ long flexible dielectric elastomer bending actuator fabricated using the methodology presented (scales bar $=1 \mathrm{~cm}$ ). A) Actuator dimensions and working principle. B) Scotch-tape test for qualitative evaluation of adhesion strength of electrode to elastomer membrane. Left: Scotch tape is placed on electrode. Right: Almost no electrode transferred when tape is removed. C) Fabricated device. Left: inactive $(0 \mathrm{~V})$, right: applied voltage $4300 \mathrm{~V}$.

due to the progressive generation of a silica-like layer [8]. This stiffening can reduce the strain output of dielectric elastomer actuator devices. The stiffening is however progressive and can therefore be minimized by reducing the plasma exposure time, but not so much as to degrade the bonding strength. The plasma exposure time of 20 seconds used for this work was empirically found to be the lowest at which repeatable bonding could be achieved.

\section{RESULTS}

We fabricate a flexible dielectric elastomer bending actuator following the design reported in [9]. The bending actuator is capable of being folded (i.e. during storage) and has been proposed as a compliant robotic gripper, and so would therefore greatly benefit from the excellent electrode adhesion (and therefore mechanical resilience) afforded by our technique.

The actuator consists of a $70 \mu \mathrm{m}$ thick prestretch PDMS (Sylgard 186, Dow Corning) membrane patterned with PDMS-carbon composite electrodes on both sides of the membrane using our technique (electrodes thickness $=4$ $\mu \mathrm{m})$. A flexible $50 \mu \mathrm{m}$ thick PET frame having several open sections is adhered to the surface of the PDMS membrane so that when the membrane prestretch is released the actuator finds equilibrium by bending. The bending angle is electromechanically modified by application of a high voltage across the electrodes (Figure 4A), generating a compressive stress, $p$, in the PDMS membrane via charge interactions, where;

$$
p=\varepsilon E^{2}
$$

where $\varepsilon$ is the permittivity of the PDMS and $E$ is the electric field across the membrane, and is equal to the applied voltage divided by the PDMS membrane thickness.

We succeed in fabricating actuators with excellent electrode adhesion as demonstrated using the "scotch-tape" test (Figure 4B). The actuator also shows a large bending angle change $\left(>90^{\circ}\right)$ upon application of a high voltage $(4.3 \mathrm{kV})$.

We next fabricate interdigitated capacitive proximity sensors with a pitch of $300 \mu \mathrm{m}$ (Figure 5A), demonstrating the resolution that can be achieved using our technique. The sensor changes its capacitance as conducting objects or insulators possessing a different permittivity than the surrounding material enters the region of the electric field generated across the electrodes [10] (Figure 5A). As the objects gets closer to the electrode surface the effect is increased leading to ability to detect proximity.

The sensor electrode is $4 \mu \mathrm{m}$ thick and is bonded to a $5 \mu \mathrm{m}$ thick PDMS membrane (MED-4901, NuSil). The sensor is encapsulated with a second $30 \mu \mathrm{m}$ thick PDMS membrane (Sylgard 186, Dow Corning) bonded by oxygen plasma activation. Electrode thickness and sheet resistance are approximately $3 \mu \mathrm{m}$ and $66 \mathrm{k} \Omega /$, respectively.

Figure $5 \mathrm{~B}$ shows the robustness of the sensor to substantial mechanical deformation. Figure 5C shows the response of the sensor to an advancing PDMS balloon (PDMS membrane thickness $5 \mu \mathrm{m}$ ) filled with deionized water, which has a significantly higher permittivity than the PDMS. The sensor reactance, inversely proportional to the capacitance, is plotted against distance of the PDMS balloon to the sensor underside (the side facing the $5 \mu \mathrm{m}$ MED-4901 membrane as shown in Figure 5A). The PDMS balloon position is controlled using a motorized stage moving and the impedance recorded using an Agilent E4980A LCR meter at $549 \mathrm{~Hz}$.

The results show that the reactance decreases by two orders of magnitude when the water bubble touches the sensor surface. Moreover, the proximity effect can clearly be seen in the range $0-1 \mathrm{~mm}$ above the sensor.

\section{CONCLUSION}

We report a novel method for the fabrication of PDMScarbon composite electrodes for silicone dielectric elastomer transducers (DETs). DETs show great potential for the development of highly deformable and damage resilient devices. Our method involves the use of laser ablation to pattern composite electrodes, which are cast on plastic substrates coated with a water-soluble sacrificial layer. We achieve bonding of the patterned electrode to PDMS silicone membranes by exposing the surfaces in oxygen plasma and placing them in contact with each other forming covalent bonds. We use our methodology to fabricate a 

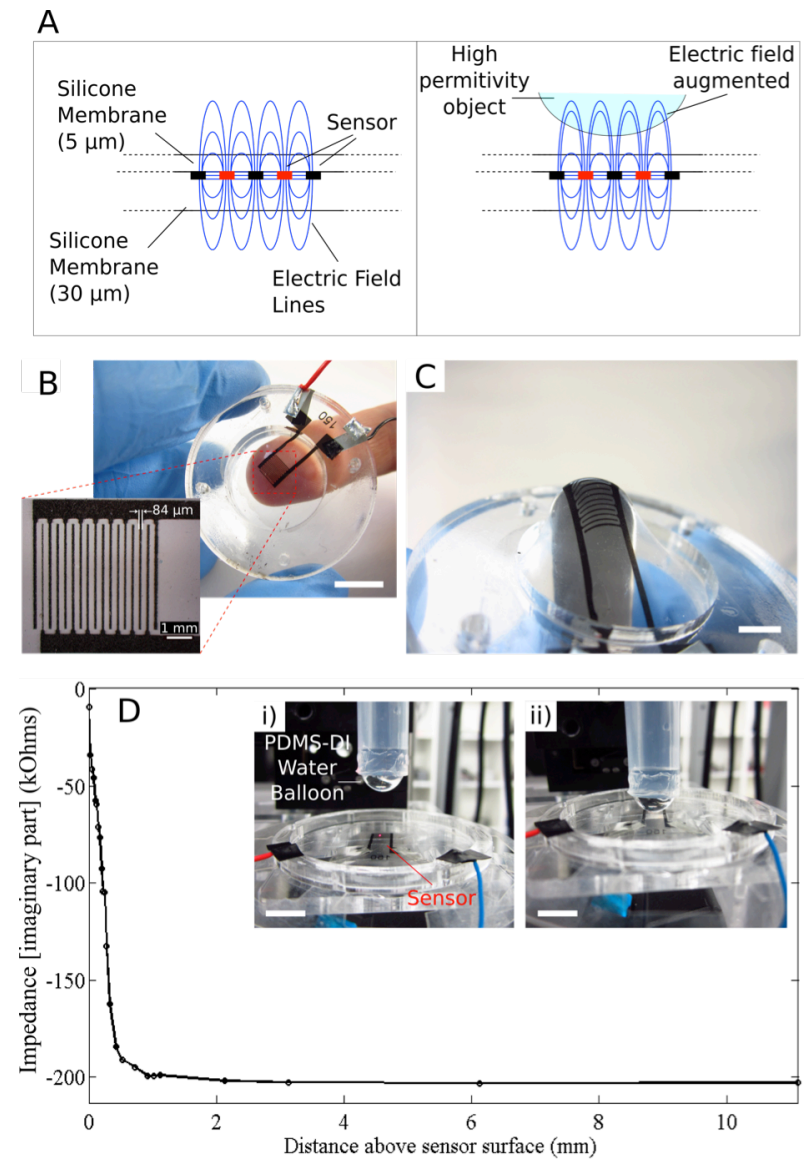

Figure 5: A) Schematic cross-section of interdigitated capacitive proximity sensor working principle. (red and black squares indicate positive and negatively changed interdigitated electrodes) Left: sensor in default state (no change in response). Right: High permittivity object penetrates and augments electric field leading to change (increase) in capacitance. B) Photograph of fabricated interdigitated capacitive proximity sensor (scale bar $=1 \mathrm{~cm}$ ) and optical microscope close-up of structure showing sub-100 $\mathrm{mm}$ features. C) Demonstration of high compliance and mechanical robustness to mechanical deformation. D) Response of capacitive proximity sensor to advancing PDMS balloon filled with deionized water. Inset figures: measurement set-up (scale bar $=1 \mathrm{~cm})$, i) start of experiment, ii) end of experiment.

$10 \mathrm{~cm}$ long flexible bending actuator and an interdigitated capacitive proximity sensor with a pitch of $300 \mu \mathrm{m}$, demonstrated its versatility. The devices show excellent electrode adhesion and mechanical robustness. The bending actuator is capable of large changes in bending angle $\left(>90^{\circ}\right)$ with the application of a high voltage, and the sensor shows excellent sensitivity and a proximity detection in the range 0-1 mm away from its surface. Hence, our process opens the door to the development of novel flexible robotic grippers or manipulators with integrated sensing capable of detecting proximity to, as well as contact with, the gripping object.

\section{ACKNOWLEDGEMENTS}

We would like to gratefully acknowledge the support of the members of the LMTS and the SAMLAB at EPFL, Nadine Besse for help with capacitive sensor design and Alexandre Poulin for use of his data-logging scripts. This work was partially funded by Swiss National Science Foundation grant \#200020_153122 and by the EPFL.

\section{REFERENCES:}

[1] Anderson, T. A. Gisby, T. G. McKay, B. M. O'Brien, and E. P. Calius, J. "Multi-functional dielectric elastomer artificial muscles for soft and smart machines,"Appl. Phys., vol. 112, no. 4, p. 041101, 2012.

[2] L. Maffli, S. Rosset, M. Ghilardi, F. Carpi, and H. Shea, "Ultrafast All-Polymer Electrically Tunable Silicone Lenses," Adv. Funct. Mater., p. n/a-n/a, 2015.

[3] S. Rosset and H. R. Shea, "Flexible and stretchable electrodes for dielectric elastomer actuators," Appl. Phys. $A$, pp. 281-307, Nov. 2012.

[4] C. Keplinger, J.-Y. Sun, C. C. Foo, P. Rothemund, "Stretchable, transparent, ionic conductors.," G. M. Whitesides, and Z. Suo, Science, vol. 341, no. 6149, pp. 984-7, Aug. 2013.

[5] P. Lotz, M. Matysek, and H. F. Schlaak, "Fabrication and Application of Miniaturized Dielectric Elastomer Stack Actuators," IEEE/ASME Trans. Mechatronics, vol. 16, no. 1, pp. 58-66, Feb. 2011.

[6] S. Bhattacharya, a. Datta, J. M. Berg, and S. Gangopadhyay, "Studies on surface wettability of poly(dimethyl) siloxane (PDMS) and glass under oxygenplasma treatment and correlation with bond strength," $J$. Microelectromechanical Syst., vol. 14, no. 3, pp. 590-597, Jun. 2005.

[7] D. B. Wolfe, J. B. Ashcom, J. C. Hwang, C. B. Schaffer, E. Mazur, and G. M. Whitesides, "Customization of Poly(dimethylsiloxane) Stamps by Micromachining Using a Femtosecond-Pulsed Laser," Adv. Mater., vol. 15, no. 1, pp. 62-65, Jan. 2003.

[8] J. Kim, "Hydrophobic Recovery of Polydimethylsiloxane Elastomer Exposed to Partial Electrical Discharge," J. Colloid Interface Sci., vol. 226, pp. 231-236, 2000.

[9] O. A. Araromi, I. Gavrilovich, J. Shintake, S. Rosset, M. Richard, V. Gass, and H. R. Shea, "Rollable Multisegment Dielectric Elastomer Minimum Energy Structures for a Deployable Microsatellite Gripper," IEEE/ASME Trans. Mechatronics, 2014.

[10] D. P. J. Cotton, I. M. Graz, and S. P. Lacour, "A multifunctional capacitive sensor for stretchable electronic skins," IEEE Sens. J., vol. 9, pp. 2008-2009, 2009.

\section{CONTACT}

O. A. Araromi, seun.araromi@epfl.ch, web:lmts.epfl.ch H. R. Shea, Herbert.shea@epfl.ch, web: 1mts.epfl.ch 